\title{
MicroRNA-92a-3p Regulates
}

Aggrecanase-1 and Aggrecanase-2

Expression in Chondrogenesis and IL-1 $\beta$ Induced Catabolism in Human Articular Chondrocytes

\author{
Guping Mao Peihui Wu Ziji Zhang $^{\mathrm{a}}$ Zhiqi Zhanga Weiming Liao ${ }^{\mathrm{a}}$ Yukang Li ${ }^{\mathrm{b}}$ \\ Yan Kang ${ }^{\text {a }}$ \\ aDepartment of Joint Surgery, First Affiliated Hospital of Sun Yat-sen University, Guangzhou, \\ Guangdong, China; ${ }^{\mathrm{b} T o w e r}$ Hill School, Wilmington, DE, USA
}

\section{Key Words}

Mirna-92a-3p • Chondrogenesis - Aggrecanase-1 - Aggrecanase-2 • Osteoarthritis • Chondrocytes

\begin{abstract}
Background/Aims: Aggrecanase-1 (ADAMTS-4) and aggrecanase-2 (ADAMTS-5) are secreted enzymes belonging to the ADAMTS (a disintegrin and metalloproteinase with thrombospondin motifs) family that play significant roles in the progression of osteoarthritis (OA). Here, we aimed to determine whether the expression of ADAMTS-4/5 in chondrogenesis and inflammation is regulated by microRNA-92a-3p (miR-92a-3p). Methods: MiR-92a-3p and ADAMTS-4/5 expressions were determined by quantitative polymerase chain reaction (qPCR). To investigate the repressive effect of miR-92a-3p on ADAMTS-4/5 expression, chondrogenic human mesenchymal stem cells (hMSCs) and human chondrocytes were transfected with mature miR-92a-3p or an antisense inhibitor (anti-miR-92a-3p), respectively. ADAMTS-4/5 protein production was quantified by enzyme-linked immunosorbent assay (ELISA), and miR$92 a-3 p$ involvement in IL-1 $\beta$-mediated catabolic effects was examined by immunoblotting. The roles of activated MAP kinases (MAPK) and nuclear factor (NF)-kB were evaluated by using specific inhibitors. Interaction between miR-92a-3p and its putative binding site in the 3'-untranslated region (3'-UTR) of ADAMTS-4/5 mRNA was confirmed by luciferase reporter assay. Results: miR-92a-3p expression was elevated in chondrogenic hMSCs, with significantly lower expression in OA cartilage than in normal cartilage. Stimulation with IL-1 $\beta$ significantly reduced miR-92a-3p expression in primary human chondrocytes (PHCs). Transfection of chondrocytes with miR-92a-3p downregulated IL-1 $\beta$-induced ADAMTS-4/5 expression, and the activity of a reporter construct containing the $3^{\prime}$-UTR of human ADAMTS-4/5 mRNA. G. Mao, P. Wu and Z. Zhang contributed equally to this study

Yukang Li

and Yan Kang

Tower Hill School, 2813 W 17th St, Wilmington, DE (USA); Department of Joint Surgery,

First Affiliated Hospital of Sun Yat-sen University, Guangzhou, Guangdong, (China);

E-Mail yukangli0418@gmail.com/neokang@163.com
\end{abstract}

KARGER 


\section{Cellular Physiology Cell Physiol Biochem 2017;44:38-52

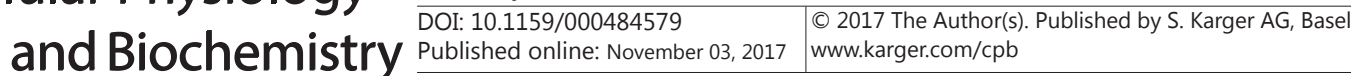 \\ Guping et al.: Role of Mir-92a-3p in Chondrogenesis and Degeneration}

MiR-92a-3p expression was suppressed upon IL-1 $\beta$-induced activation of MAPK and NF-KB in chondrocytes. Conclusion: MiR-92a-3p is an important regulator of ADAMTS-4/5 in human chondrocytes and may contribute to the development of OA.

(C) 2017 The Author(s)

Published by S. Karger AG, Basel

\section{Introduction}

Osteoarthritis $(\mathrm{OA})$ is usually a progressive disease of the synovial joints that represents the failed repair of joint damage and ultimately results in the breakdown of cartilage and bone, leading to symptoms of pain, stiffness, and functional disability [1-3] It is widely accepted that the progression of $\mathrm{OA}$ is associated with proinflammatory cytokines derived from chondrocytes, such as interleukin (IL)-1 $\beta$ and tumor necrosis factor- $\alpha$ [4]. IL-1 $\beta$ activity can disrupt chondrocyte anabolism homeostasis via repressing the synthesis of proteoglycan and collagen [5, 6]. Proteoglycan and collagen degradation are principally carried out by members of the matrix metalloproteinase (MMP) family, such as MMP3, MMP7, and MMP13, which play important roles in degrading cartilage collagens [7-9]. However, previous biochemical studies have demonstrated that members of the a disintegrin and metalloproteinase with thrombospondin motifs (ADAMTS) family such as ADAMTS-1, -4 , $-5,-8,-9$, and -15 possess aggrecanase activity and can induce the degradation of cartilage proteoglycan-aggrecan in OA [10-14]. Knockdown of ADAMTS-4/5 exerted protective effects against proteoglycan degradation and attenuated the severity of murine OA [15], and the degradation of aggrecan was decreased when ADAMTS-4/5 small interfering RNA was used in human cartilage stimulated by IL-1 $\beta$ [16]. This finding suggested that both ADAMTS-4 and ADAMTS-5 are responsible for the aggrecan degradation in OA in humans. In support of this idea, recent studies have demonstrated that ADAMTS-4/5 are overexpressed in human OA cartilage, with a positive correlation with the degree of cartilage destruction [11, 17 20]. These results suggest that ADAMTS- $4 / 5$ play a pivotal role in human OA cartilage and that their induction contributes to the development of OA. Aggrecanases are thus potential targets for the development of disease-modifying OA drugs (DMOADs).

MicroRNAs (miRNAs) are short endogenous oligonucleotides $(\sim 22 \mathrm{bp})$ that play a significant role in the regulation of post-transcriptional gene expression. MiRNAs regulate target gene expression by repressing mRNA translation and accelerating mRNA degradation $[21,22]$. An increasing number of reports have demonstrated that miRNAs regulate chondrogenesis and cartilage development. For example, miR-445-3p regulates chondrogenic ATDC5 cells via inhibiting Runx2[23] and modulates cartilage development and degeneration through modification of histone H3 acetylation [24]. Moreover, miR-125b [18] and miR-140[20, 25] regulate ADAMTS-4 or ADAMTS-5 expression and the IL-1 $\beta$ induced catabolic response in human chondrocytes, respectively.

We previously reported a 4.4-fold increase in miR-92a-3p expression when human adipose-derived stem cells were induced to differentiate into chondrocytes [26]. We also observed the rapid up-regulation of miR-92a-3p in chondrogenic human mesenchymal stem cells (hMSCs) beginning on day 7, which peaked at 21 days and was followed by a marked decrease in expression levels at day 28[27]. In addition, the expression level of miR-92a-3p was significantly reduced in $\mathrm{OA}$ cartilage compared with that detected in normal cartilage [27]. In a luciferase reporter assay, we demonstrated that histone deacetylase 2 (HDAC2) was the target of miR-92a-3p [27]. However, using miRNA target prediction algorithms, we discovered that ADAMTS- 4 and ADAMTS- 5 may be potential targets of miR-92a-3p. Since miR-92a-3p and ADAMTS-4/5 are essential for mediating chondrocyte development and cartilage degeneration, we hypothesized that miR-92a-3p participates in OA pathogenesis by targeting and suppressing ADAMTS-4/5 expression.

To test this hypothesis, in the present study, we attempted to validate the regulation and molecular mechanism of miR-92a-3p in human chondrocytes. In particular, to investigate the repressive effect of miR-92a-3p on ADAMTS- $4 / 5$ expression, chondrogenic hMSCs and human chondrocytes were transfected with mature miR-92a-3p or an antisense inhibitor 


\section{Cellular Physiology Cell Physiol Biochem 2017;44:38-52

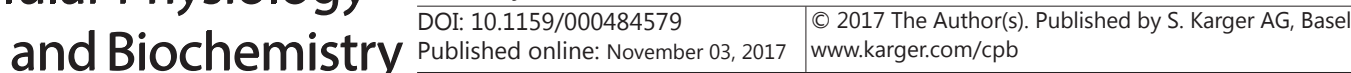 \\ Guping et al.: Role of Mir-92a-3p in Chondrogenesis and Degeneration}

(anti-miR-92a-3p), respectively, and the potential involvement of miR-92a-3p in IL-1 $\beta$ mediated catabolic effects was examined. Furthermore, we examined the potential molecular mechanism underlying this regulation by exploring the roles of activated mitogen-activated protein kinase (MAPK) and nuclear factor (NF)- $\mathrm{BB}$ using specific inhibitors, and determined the interaction between miR-92a-3p and its putative binding site in the $3^{\prime}$-untranslated regions (3'-UTRs) of ADAMTS-4/5 mRNA with a luciferase reporter assay. These findings might highlight miR-92a-3p as a novel regulator of cartilage homeostasis, indicating a candidate therapeutic target for $\mathrm{OA}$.

\section{Materials and Methods}

This study adhered to the standards of the Ethics committee on Human Experimentation at the First Affiliated Hospital of Sun Yat-Sen University, China (IRB: 2011011) and the Helsinki Declaration (2000). All volunteers provided written informed consent.

\section{Culture of $h M S C$}

Bone marrow samples were harvested by iliac crest aspiration from six normal human donors (mean age: 37 years, range: 35-39 years, male: 3, female: 3). Density gradient centrifugation methods were used to isolate hMSCs [28]. In brief, the bone marrow was rinsed several times with sterile phosphate-buffered saline (PBS) before addition of Ficoll-Paque ${ }^{\mathrm{TM}}$ PLUS (GE Healthcare, Uppsala, Sweden), and centrifuged for $18 \mathrm{~min}$ at $1000 \times \mathrm{g}$. The interfacial mononuclear cells were collected and resuspended in hMSC basal medium (alpha-modified Eagle's medium; Gibco Life Technology Grand Island, NY, USA) supplemented with 10\% fetal bovine serum (FBS; Gibco Life Technology), and 1\% penicillin/streptomycin (Gibco Life Technology). Cells from bone marrow aspirates were seeded at a density of approximately 50, 000 nucleated cells $/ \mathrm{cm}^{2}$ in a culture flask. The cells were cultured at $37^{\circ} \mathrm{C}$ in an atmosphere of $5 \% \mathrm{CO}_{2}$. The medium was changed every 3 days. When the cultures were near $80 \%$ confluence, the cells were trypsinized with $0.05 \%$ trypsin/ ethylenediaminetetraacetic acid and passaged (1:2) in culture. The medium was refreshed every 3 days, and expanded cells at passage 3 or 5 were used for the experiments.

\section{Chondrogenesis in a micromass culture}

For micromass culturing, the cells were resuspended in incomplete chondrogenic induction medium ( $97 \mathrm{~mL}$ of hMSC chondrogenic differentiation basal medium, $10 \mu \mathrm{L}$ of dexamethasone, $300 \mu \mathrm{L}$ of ascorbate, I mL of ITS+ [insulin, transferrin, and selenium; BD Biosciences, Franklin Lakes, NJ, USA] supplement, 100 $\mu \mathrm{L}$ of sodium pyruvate, and $100 \mu \mathrm{L}$ of proline; Cyagen, Guangzhou, China) at a specified density of $10^{5}$ cells/ $\mu \mathrm{L}$ of medium, and droplets $(12.5 \mu \mathrm{L})$ were then carefully added to each well of a 24 -well plate. The hMSCs were allowed to adhere at $37^{\circ} \mathrm{C}$ for $70 \mathrm{~min}$. Then, they were divided into two groups. The first group was cultured in incomplete chondrogenic induction medium and the second group was cultured in complete chondrogenic induction medium $(10 \mu \mathrm{L}$ of TGF- $\beta 3$ was added to convert $1 \mathrm{~mL}$ of incomplete chondrogenic medium into complete medium; Cyagen); the medium was replaced every 3 days [29].

\section{Primary chondrocyte collection, isolation, and cell culture}

Cartilage tissues were harvested from OA knee joints during total knee replacement operation from patients ( $\mathrm{n}=8$; mean \pm standard deviation [SD] age: $65.8 \pm 2.24$ years; male: 4 , female: 4 ). Normal cartilage tissues were collected from patients $(n=8$; mean \pm SD age: $64.4 \pm 2.86$ years; male: 4 , female: 4$)$ with no previous history of OA or RA, who underwent total hip replacement surgery because of fractures of the femoral neck. OA cartilage was collected from gross erosion areas, while normal cartilage was collected from areas with no surface irregularity. PHCs were isolated as described previously [30, 31]. The isolated chondrocytes were seeded in $75-\mathrm{cm}^{2}$ culture flasks and incubated in Dulbecco's modified Eagle's medium/ F12 (Gibco Life Technology) containing 5\% FBS, 2\% penicillin and streptomycin, and ITS+ premix. During the culture period, cells were incubated at $37^{\circ} \mathrm{C}$ in a humidified atmosphere of $5 \% \mathrm{CO}_{2}$. The medium was changed every 2 days. 


\section{Cellular Physiology Cell Physiol Biochem 2017;44:38-52

 \\ Guping et al.: Role of Mir-92a-3p in Chondrogenesis and Degeneration}

RNA extraction, reverse transcription, and $q R T-P C R$

Total RNA was extracted with a miRNeasy Mini Kit (QIAGEN, CA, USA) following the manufacturer's instructions. The concentration and purity of the extracted RNA were analyzed using an Epoch Multi-Volume Spectrophotometer System (BioTek Instruments, Inc., Winooski, VT, USA). cDNA was obtained using the PrimeScript $(\AA$ miRNA cDNA Synthesis Kit

Table 1. Primers for quantitative real-time polymerase chain reaction

(TAKARA Biotechnology, Japan)

\begin{tabular}{llc}
\hline Gene & & primer sequence (5'-3') \\
\hline hsa-ADAMTS4 & F & GGTCAAGGTCCCATGTGCAAC \\
hsa-ADAMTS4 & R & GAATGCGGCCATCTTGTCATC \\
hsa-ADAMTS5 & F & GGCCTCCATCGCCAATAGG \\
hsa-ADAMTS5 & R & GGATAGCTGCATCGTAGTGCT \\
hsa-Aggrecan & F & GATGTTCCCTGCAATTACCACCTC \\
hsa-Aggrecan & R & TGATCTCATACCGGTCCTTCTTCTG \\
hsa-GAPDH & F & GCACCGTCAAGGCTGAGAAC \\
hsa-GAPDH & R & TGGTGAAGACGCCAGTGGA \\
hsa-U6 & F & CTCGCTTCGGCAGCACA \\
hsa-U6 & R & AACGCTTCACGAATTTGCGT \\
hsa-miR-92a-3p & F & CACTTGTCCCGGCCTGTAAA \\
\hline
\end{tabular}
following the manufacturer's instructions. qRT-PCR was performed using SYBR@ Premix Ex Taq ${ }^{\mathrm{TM}}$ II (TAKARA Biotechnology) according to the manufacturer's instructions on a CFX96 system. The specific primers are listed in Table 1 . The relative gene expression levels were calculated using the $2^{-\Delta \Delta C t}$ method. All experiments were performed at least in triplicate.

\section{Transfection}

The hMSCs and PHCs were transfected with an agomir or antagomir (RiboBio, Guangzhou, China) of miR-92a-3p at a concentration of $50 \mathrm{nM}$ or $100 \mathrm{nM}$. Lipofectamine ${ }^{\circledR} 2000$ Transfection Reagent (Gibco Life Technologies) was used to transfect cells according to the manufacturer's instructions. Non-specific microRNA (miR-Control; RiboBio) was used as a control.

\section{Immunohistochemical analysis}

Immunohistochemical analysis was performed as described previously [32]. Formalin-fixed and paraffin-embedded cartilage specimens were sectioned and stained with Safranin 0 for histological evaluation. In brief, sections were incubated for $10 \mathrm{~min}$ with $50 \mathrm{~mL}$ of $3 \% \mathrm{H}_{2} \mathrm{O}_{2}$ solution, and then incubated with primary anti-human ADAMTS-4 (1:200 dilution; Abcam, Cambridge, MA, USA) or ADAMTS-5 antibody (1:50; Santa Cruz Biotechnology, Inc., Santa Cruz, CA) overnight at $4^{\circ} \mathrm{C}$. Negative controls were prepared by substituting PBS for the primary antibody. The sections were then incubated with the secondary antibody horseradish peroxidase-conjugated anti-rabbit IgG (Cell Signaling Technology, Boston, USA) for $30 \mathrm{~min}$

\section{Western blot analysis}

Western blotting was carried out as described previously [23]. Proteins were resolved on a $10 \%$ sodium dodecyl sulfate-polyacrylamide gel and transferred to polyvinylidene difluoride membranes (Millipore, Bedford USA). After blocking with 5\% non-fat milk in Tris-buffered saline plus 0.1\% Tween 20, the membranes were incubated with primary antibodies against ADAMTS-4 (1:1, 000; Abcam), ADAMTS-5 (1:200; Santa Cruz Biotechnology, Inc.), aggrecan (1:1, 000; Millipore), and $\beta$-actin (1:3, 000; Cell Signaling Technology). After washing, the blots were incubated with the corresponding secondary antibodies (Cell Signaling Technology) at room temperature for $1 \mathrm{~h}$ and visualized using an ECL chemiluminescence kit (Millipore).

\section{ADAMTS-4 and ADAMTS-5 ELISA}

Cells were stimulated with IL- $1 \beta$ for $24 \mathrm{~h}$, and the ADAMTS- 4 and ADAMTS- 5 protein levels in the culture supernatants were quantified by ELISA using ADAMTS-4 and ADAMTS-5 immunoassay kits, according to the manufacturer's instructions (CusaBio, Wuhan, China). Plates were read at $450 \mathrm{~nm}$ using an HT Microplate Reader (BioTek, Winooski, VT, USA), and the ADAMTS-4 and ADAMTS-5 concentrations in the samples were calculated using a standard curve. 


\section{Cellular Physiology Cell Physiol Biochem 2017;44:38-52

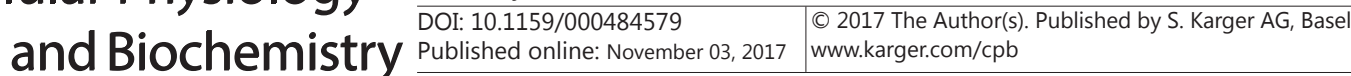 \\ Guping et al.: Role of Mir-92a-3p in Chondrogenesis and Degeneration}

Luciferase constructs and reporter assay

The pmiR-RB-REPORT ${ }^{\mathrm{TM}}$ luciferase vector (OBIO, Shanghai, China) was used for dual luciferase assays. The $3^{\prime}$-UTR fragments containing the predicted seed sequences were inserted into the vector using the Xhol/NotI restriction sites. The $3^{\prime}$-UTR fragments of the ADAMTS- $4 / 5$ coding sequences were PCR-amplified using the following primers: hsa-ADAMTS-4-3'-UTR-F, 5' -ATAGGCCGGCATAGACGCGTCCTCACTATCCCGGCTGC-3', hsa-ADAMTS-4-3'-UTR-R, 5'- AAAGATCCTTTATTAAGCTTTGAGCACTTACTAGATGCTGAGTG-3'; hsa-ADAMTS-5(site1)-3'-UTR-F， 5' -ATAGGCCGGCATAGACGCGTCCTGTGGTTATGATCTTATGCAC-3'， hsaADAMTS-5(site1)-3'-UTR-R, 5' - AAAGATCCTTTATTAAGCTTCAGTCTTATTAATATTAACCACTTTTTG-3'; hsa-ADAMTS-5(site2)-3'-UTR-F， 5'- ATAGGCCGGCATAGACGCGTCAACTTAACTGGCTAGTACATTG-3', hsaADAMTS-5(site2)-3'-UTR-R, 5'- AAAGATCCTTTATTAAGCTTTACTTTAACCTAGTTTACAATTTATATTTATTATG-3'. The following primers were used for the mutated (mut) sequences: hsa-ADAMTS-4-mut-F, 5'ATTGTGCACGATGTATCCAGCCTGGGCGACAG-3', hsa-ADAMTS-4-mut-R: 5'- CTGGATACATCGTGCACAATCTCGGCTCACTG-3'; hsa-ADAMTS-5(site1)-mut-F, 5'-ATAACTCCCAAGTTTTTCTAGCGACGTTGGTCACCACTGTCACGTGAAG-3'; hsa-ADAMTS-5(site1)-mut-R, 5'-CCAACGTCGCTAGAAAAACTTGGGAGTTATTGAAC-3'; hsa-ADAMTS-5(site2)-mut-F, 5'-AATAACATAAATGCGACGTTAGCTTGAAAAAAAATCAGGCC-3'; and hsa-ADAMTS-5(site2)-mut-R, 5'-AACGTCGCATTTATGTTATTGGTAAACACTTGGTG-3'. The resulting PCR products were digested and cloned into the pmiRs-RB-REPORT ${ }^{\mathrm{TM}}$ vector. For the dual luciferase assay, $1.2 \times 10^{4}$ cells (HEK293) in a 96-well plate were transfected with $50 \mathrm{nM}$ miR-92a-3p or miR-NC (RibioBio) and $100 \mathrm{ng}$ of vector. Luciferase activity was measured at $48 \mathrm{~h}$ post-transfection using the Dual-Luciferase ${ }^{\circledR}$ Reporter Assay System (Promega, Madison, WI, USA). Firefly luciferase activity was then normalized to the corresponding Renilla luciferase activity.

\section{Statistical analysis}

All of the experiments were performed with at least three biological replicates. Data were evaluated as the mean \pm SD. The Student t-test and Mann-Whitney U test were used to identify differences between groups as appropriate. $P<0.05$ was considered statistically significant [33]. All analyses were performed using IBM SPSS Version 20 (IBM Corporation, Armonk, NY, USA).

\section{Results}

Expression patterns of ADAMTS-4/5 during the chondrogenic differentiation of hMSCs

To investigate the expression patterns of ADAMTS-4 and ADAMTS-5 during chondrogenesis, hMSCs were induced to differentiate into chondrocytes in vitro with transforming growth factor (TGF)- $\beta 3$. ADAMTS- $4 / 5$ expression was significantly upregulated between days 21 and 35, (Fig. 1a A, B). The expression of the chondrogenic marker aggrecan was first detected on day 7 , which peaked at 21 days, followed by a marked decrease in expression at day 28 (Fig. 1a C).

miR-92a-3p regulates the expression of ADAMTS-4/5 in hMSCs during chondrogenesis

To further investigate whether miR-92a-3p regulates ADAMTS-4/5 expression during chondrogenesis, miR-92a-3p was overexpressed or inhibited in the hMSCs. Monolayered hMSCs were transfected with either anti-miR-92a-3p or miR-92a-3p twice, one day after plating and again after 3 days, and were then induced to undergo chondrogenesis for 14 days (Fig. 1b). Overexpression of miR-92a-3p significantly decreased the ADAMTS-4/5 expression level (Fig. 1b A, B and D), and increased the aggrecan expression level (Fig. 1b C and D). In contrast, the miR-92a-3p inhibitor markedly upregulated ADAMTS-4/5 expression (Fig.1b E, $\mathrm{F}$, and $\mathrm{H}$ ) and downregulated aggrecan expression (Fig. 1b G and $\mathrm{H}$ ).

\section{Expression of ADAMTS-4/5 in normal and OA cartilage}

To assess the potential involvement of ADAMTS-4/5 in the progression of OA, we compared its expression level in normal and OA cartilage samples. Compared with normal cartilage, OA cartilage exhibited higher levels of ADAMTS-4/5 mRNA (Fig. 2A and B) and protein (Fig. 2C and D), as assessed by quantitative reverse transcription-polymerase chain reaction (qRT-PCR) and immunohistochemical analysis, respectively. 


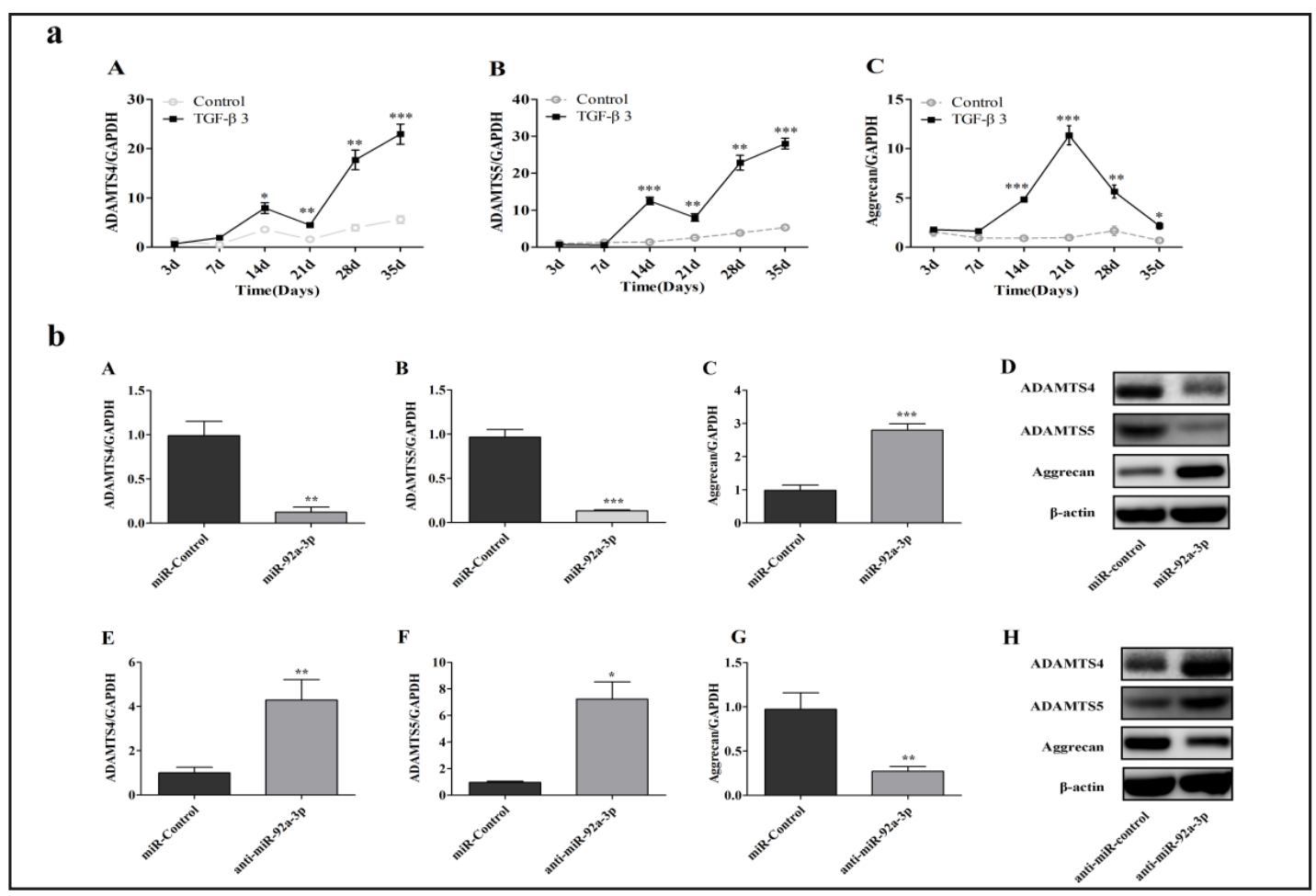

Fig. 1. Expression of miR-92a-3p and ADAMTS-4 and ADAMTS-5 during chondrogenesis. a. hMSCs were induced to undergo chondrogenesis with TGF- $\beta 3$ for $3,7,14,21,28$, and 35 days as indicated (solid lines). Gene expression levels of ADAMTS-4 (A), ADAMTS-5 (B), and aggrecan (C) were determined by qRT-PCR. b. MiR-92a-3p regulates the expression of ADAMTS-4, ADAMTS-5 and aggrecan during chondrogenesis. hMSCs were transfected with miR-92a-3p or miR-control and anti-miR-92a-3p or anti-miR-control, and then treated with TGF- $\beta 3$ to induce chondrogenesis. The expression levels of ADAMTS-4 (A, E), ADAMTS-5 (B, F), and aggrecan (C, G) were estimated by qRT-PCR and western blotting (D, H). HMSCs cultured without TGF- $\beta 3$ at the corresponding time points served as negative controls (broken lines)GAPDH was used as an endogenous control in qRT-PCR, and $\beta$-actin was used as the internal control in western blotting. Data are presented as the means \pm standard deviation of six samples. ${ }^{*} \mathrm{P}<0.05 ;{ }^{* *} \mathrm{P}<0.01 ;{ }^{* * *} \mathrm{P}<0.001$ vs. negative controls. Abbreviations: hMSCs, human mesenchymal stem cells; ADAMTS4, ADAM metallopeptidase with thrombospondin type 1 motif, 4; ADAMTS5, ADAM metallopeptidase with thrombospondin type 1 motif, 5; qRT-PCR, quantitative reverse-transcription polymerase chain reaction.

Fig. 2. Expression of ADAMTS-4 and ADAMTS-5 in normal and osteoarthritis (OA) cartilage. The relative ADAMTS-4/5 mRNA levels in normal and OA cartilages were determined by SYBR ${ }^{\circledR}$ green-based qRT-PCR (A and B). Each dot represents a value from a single experiment of one donor. The bars show the mean and 95\% confidence intervals of the values from four different donors per group. ADAMTS-4/5 protein levels were determined in normal cartilage and OA cartilage by immunohistochemistry (C, D). Data shown are representative of results

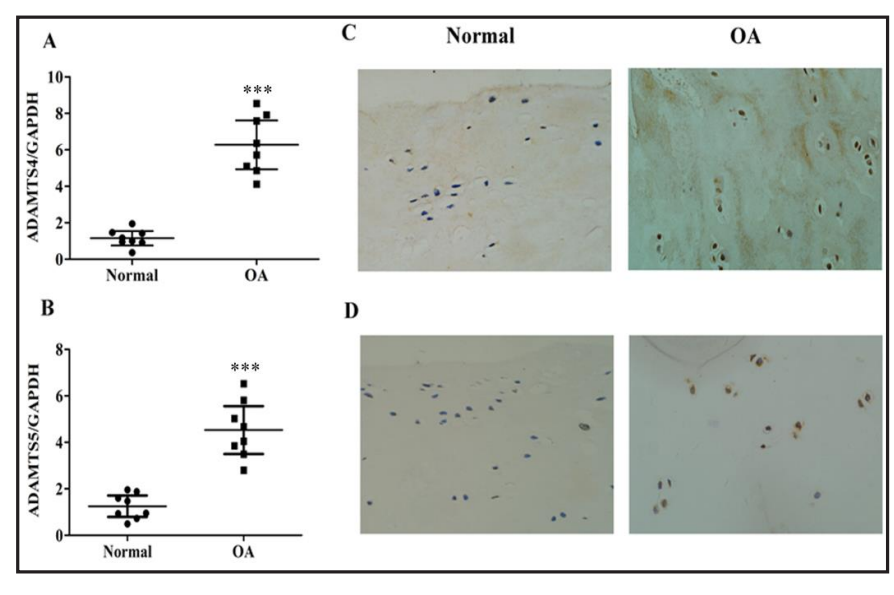
from four normal and $\mathrm{OA}$ cartilages (magnification, $\times 400$ ). ${ }^{* * *} \mathrm{P}<0.001$ vs. negative controls. Abbreviations: ADAMTS4, ADAM metallopeptidase with thrombospondin type 1 motif, 4; ADAMTS5, ADAM metallopeptidase with thrombospondin type 1 motif, 5; qRT-PCR, quantitative reverse-transcription polymerase chain reaction. 
Opposing expression between ADAMTS-4/5 and miR-92a-3p by IL-1 $\beta$ stimulation in human chondrocytes

Next, we evaluated the regulation of miR-92a-3p expression via IL-1 $\beta$. Stimulation of chondrocytes with IL-1 $\beta$ resulted in decreased miR-92a-3p expression (Fig. $3 \mathrm{~A}$ and D), and increased levels of ADAMTS-4/5 mRNA (Fig. 3B, C, E, and F) and protein (Fig. 3G, H, I, and J) in a dose- and time-dependent manner. These results suggested that the reduced miR-92a$3 p$ expression in response to IL-1 $\beta$ was correlated with increased ADAMTS-4/5 expression.

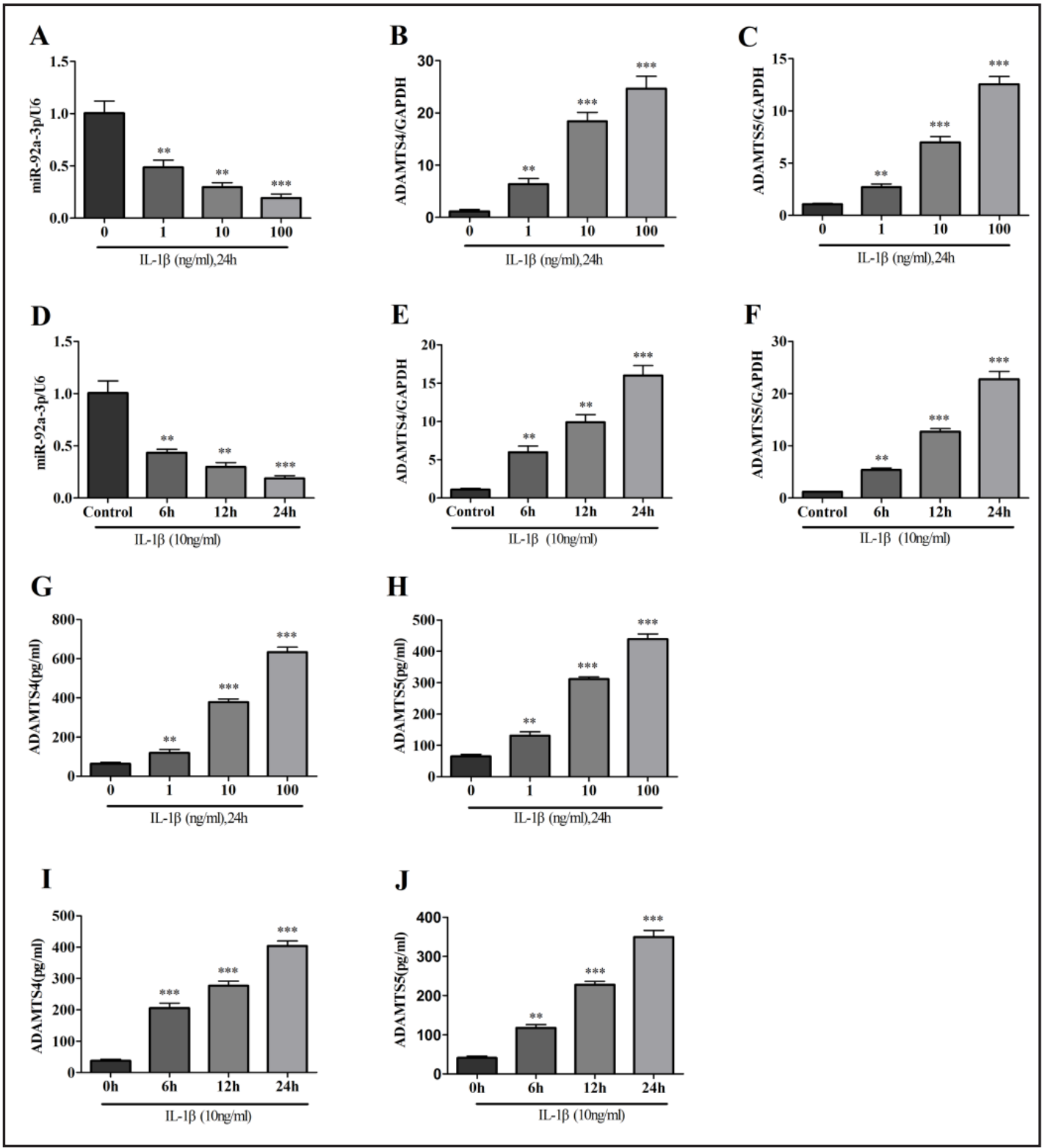

Fig. 3. Expression of miR-92a-3p and ADAMTS-4/5 in IL-1 $\beta$-stimulated human chondrocytes. Primary human chondrocytes were deprived of serum for $6 \mathrm{~h}$, and then left untreated or treated with various concentrations of IL-1 $\beta$ for the indicated times (A-J). The relative expression levels of miR-92a-3p (A, D), ADAMTS-4 (B, E), and ADAMTS-5 (C, F) were assessed by qRT-PCR; U6 and GAPDH were used as endogenous controls. IL-1 $\beta$-induced ADAMTS-4/5 production in primary human chondrocytes (PHCs) was determined by enzyme-linked immunosorbent assay after 6, 12, and $24 \mathrm{~h}(\mathrm{G}, \mathrm{I}, \mathrm{H}, \mathrm{J})$ of stimulation with IL-1 $\beta$. Data are presented as means \pm standard deviation of four samples. ${ }^{*} P<0.05$; ${ }^{* *} P<0.01$; ${ }^{* * *} P<0.001$ vs. negative controls. 


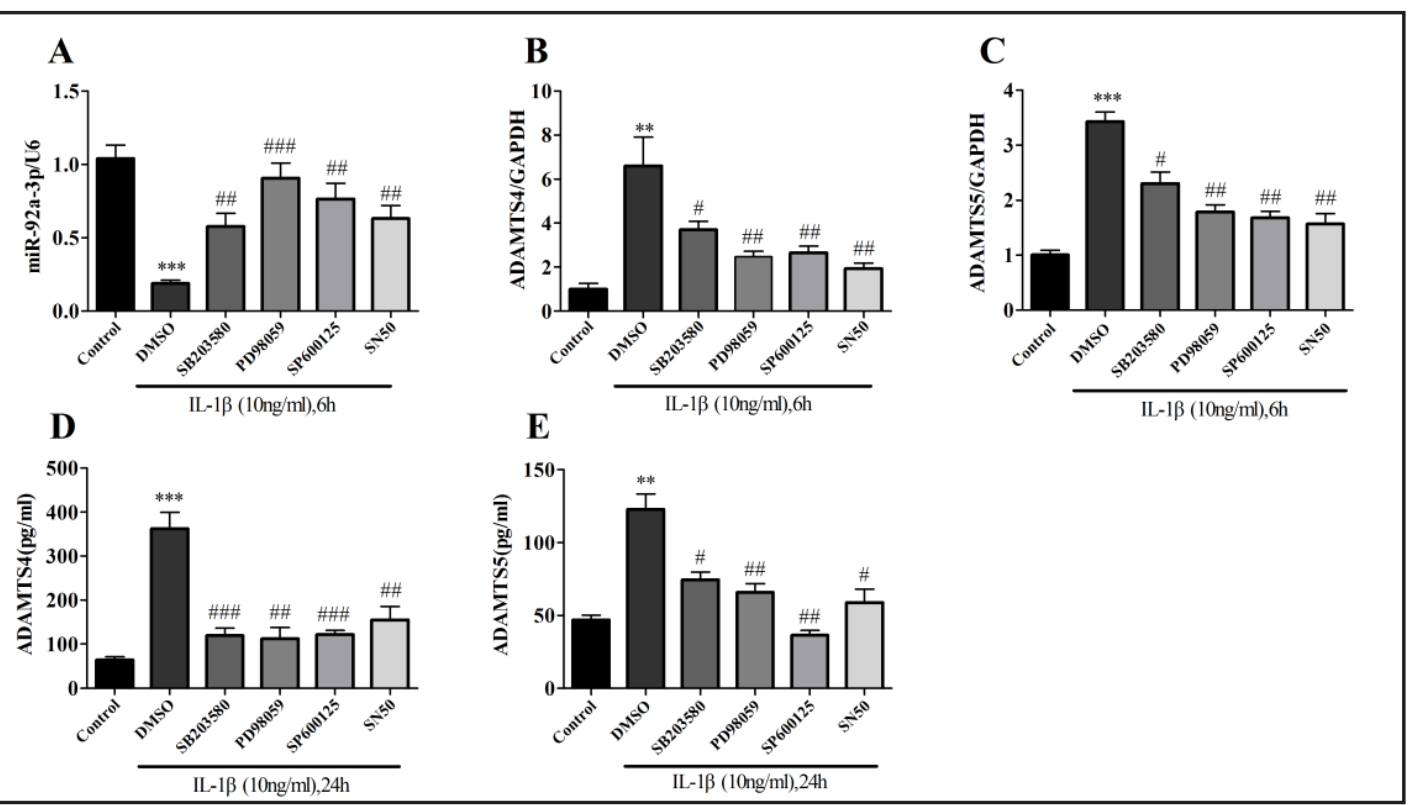

Fig. 4. Negative correlation between the expression patterns of miR-92a-3p and ADAMTS- $4 / 5$ regulated by IL-1 $\beta$-induced signaling pathways. PHCs were pretreated for $2 \mathrm{~h}$ with MAPK inhibitors (SB203580, PD98059, or SP600125) or an NF- KB inhibitor (SN50), and then left unstimulated or stimulated with IL$1 \beta$ for $6 \mathrm{~h}(\mathrm{~A}, \mathrm{~B}, \mathrm{C})$ or $24 \mathrm{~h}$ (D, E). PHCs were analyzed by qRT-PCR for miR-92a-3p (A) and ADAMTS-4/5 mRNA expression (B, C), and by ELISA for ADAMTS-4/5 protein production in culture supernatants (D, E). U6 and GAPDH were used as endogenous controls for qRT-PCR. Data are presented as means \pm standard deviation of four samples. P-values were computed vs. non-stimulated controls $\left({ }^{*} \mathrm{P}<0.05 ;{ }^{*} \mathrm{P}<0.01\right.$; ${ }^{* * *}$ $\mathrm{P}<0.001$ ) or IL-1 $\beta$-stimulated controls (\#P<0.05; \#\#P<0.01; \#\#\# $\mathrm{P}<0.001$ ). Abbreviations: ADAMTS4, ADAM metallopeptidase with thrombospondin type 1 motif, 4; ADAMTS5, ADAM metallopeptidase with thrombospondin type 1 motif, 5; qRT-PCR, quantitative reverse-transcription polymerase chain reaction; ELISA, enzyme-linked immunosorbent assay.

Role of IL-1 $\beta$ signaling pathways in the regulation of miR-92a-3p and ADAMTS-4/5 expression in human chondrocytes

To investigate which signaling pathways of IL-1 $\beta$ regulate the expression of miR-92a-3p and ADAMTS- $4 / 5$, human chondrocytes were pretreated for $2 \mathrm{~h}$ with the NF- $\kappa B$ inhibitor SN50 or various MAPK inhibitors such as the MEK-1/2 inhibitor PD98059, p38 MAPK inhibitor SB203580, and JNK inhibitor SP600125, and then stimulated with IL-1 $\beta$ for $6 \mathrm{~h}$ (Fig. 4A, B, and C) or $24 \mathrm{~h}$ (Fig. 4D and E). Compared with the expression levels in unstimulated controls, miR-92a-3p expression in IL-1 $\beta$-stimulated primary human chondrocytes (PHCs) was decreased by $70.4 \%$, while pretreatment with NF- $\mathrm{BB}$ and MAPK inhibitors attenuated the inhibitory effects of IL-1 $\beta$ on miR-92a-3p expression (Fig. 4A). IL-1 $\beta$ stimulation induced an obvious increase in ADAMTS-4/5 mRNA and protein expression, while pretreatment with NF- $\kappa B$ and MAPK inhibitors suppressed the IL- $1 \beta$-induced ADAMTS- $4 / 5$ expression in PHCs (Fig. 4B-E). These results indicated that the IL-1 $\beta$-stimulated activation of NF- $\kappa B$ and MAPK signaling pathways might be necessary for the suppressive effect of IL-1 $1 \beta$ on miR-92a-3p expression in PHCs.

Negative regulation of ADAMTS-4/5 expression by miR-92a-3p in humanchondrocytes

To verify whether IL-1 $\beta$-responsive miR-92a-3p regulates ADAMTS- $4 / 5$ expression, PHCs were transfected with miR-92a-3p, miR-control, anti-miR-92a-3p, or anti-miR-control. Forty-eight hours after transfection, the cells were stimulated with IL-1 $\beta$ for 24 h. ADAMTS- $4 / 5$ mRNA expression was estimated by qRT-PCR, while ADAMTS-4/5 protein expression was estimated by enzyme-linked immunosorbent assay (ELISA) and western blotting. 
Fig. 5. Effect of miR-92a-3p on IL$1 \beta$-induced ADAMTS-4/5 expression. PHCs were transfected with miR92a-3p or non-specific control microRNA (miR-Control), and with anti-miR-92a-3p or anti-miR-Control, and then left unstimulated or stimulated with IL-1 $\beta$ for $24 \mathrm{~h}$ for qRT-PCR analysis respectively. Forty-eight hours after transfection with miR-92a-3p or miR-Control (A, B, C, $G, H)$, or with antimiR-92a-3p or antimiR-Control (D, E, F, I, J), the expression levels of miR-92a-3p (A, D), ADAMTS-4 (B, E), and ADAMTS-5 (C, F) were determined by qRT-PCR, and ADAMTS-4/5 protein production (G-J) in culture supernatants were determined by ELISA, respectively. U6 and GAPDH were used as internal controls for microRNA and mRNA analysis, respectively. Data are presented as
D

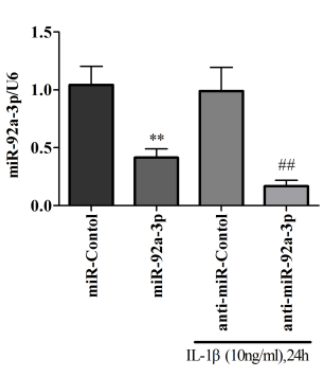

G
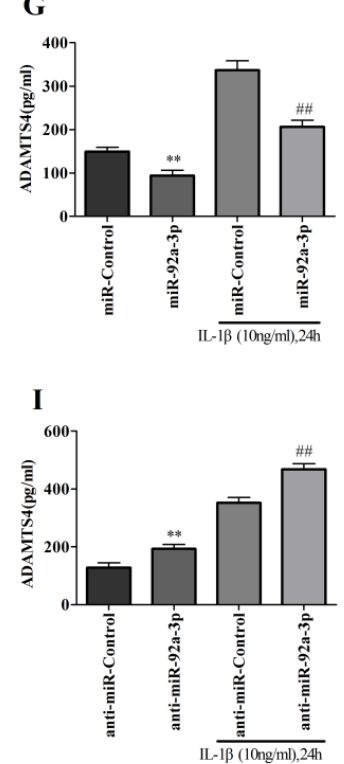

H
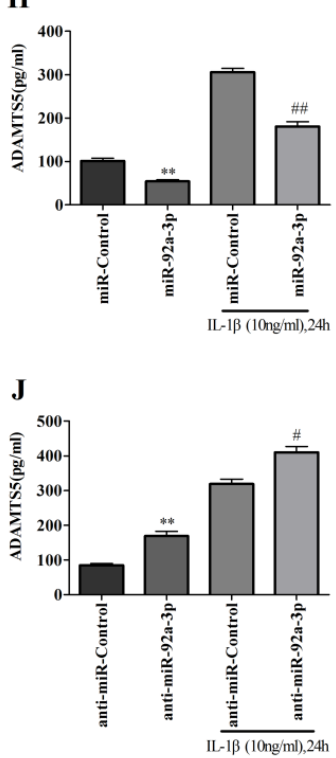
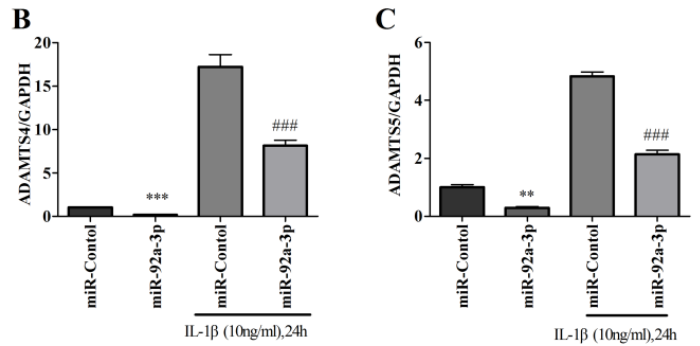

E
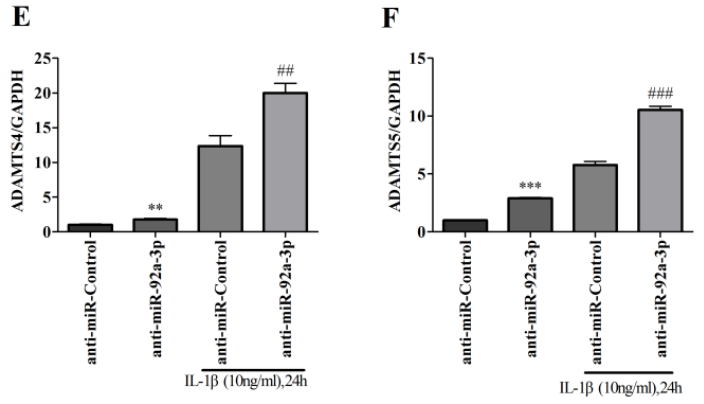

means \pm standard deviation of four samples. P-values were computed vs. non-stimulated controls $\left({ }^{*} \mathrm{P}<0.05\right.$; ${ }^{* *} \mathrm{P}<0.01$; $\left.{ }^{* * *} \mathrm{P}<0.001\right)$ or IL-1 $\beta$-stimulated controls ( $\mathrm{P}<0.05$; \#\# $<<0.01$; \#\#\# $\left.\mathrm{P}<0.001\right)$. Abbreviations: ADAMTS4, ADAM metallopeptidase with thrombospondin type 1 motif, 4; ADAMTS5, ADAM metallopeptidase with thrombospondin type 1 motif, 5; qRT-PCR, quantitative reverse-transcription polymerase chain reaction; ELISA, enzyme-linked immunosorbent assay. Abbreviations: ADAMTS4, ADAM metallopeptidase with thrombospondin type 1 motif, 4; ADAMTS5, ADAM metallopeptidase with thrombospondin type 1 motif, 5.

Overexpression of miR-92a-3p in PHCs significantly decreased the IL-1 $\beta$-induced expression of ADAMTS-4/5 mRNA (Fig. 5B and C), while suppression of miR-92a-3p expression with anti-miR-92a-3p led to a significant increase in ADAMTS-4/5 mRNA expression (Fig. 5E and F). In addition, overexpression of miR-92a-3p significantly decreased the IL- $1 \beta$-induced production of ADAMTS-4/5 protein, as estimated by ELISA and western blotting (Fig. 5G, H, and Fig.6A), while transfection with anti-miR-92a-3p significantly upregulated ADAMTS-4/5 
Fig. 6. Effect of miR-92a-3p on I L - $1 \beta$-induced A D A M T S - 4 / 5 protein expression. PHCs were transfected with miR-92a-3p or non-specific control microRNA (miR-Control), and with antimiR-92a-3p or anti-miR-Control, and then left unstimu-

lated or stimulated with IL-1 $\beta$ for $24 \mathrm{~h}$. ADAMTS-4/5 protein level was analyzed by western blot (A and B).Abbreviations: ADAMTS4, ADAM metallopeptidase with thrombospondin type 1 motif, 4; ADAMTS5, ADAM metallopeptidase with thrombospondin type 1 motif, 5;

Fig. 7 miR-92a- a $3 p$ directly $\mathrm{t}$ a r g e t s ADAMTS-4 and $\mathrm{AD}$ A M T S - 5 . a. Alignment of the nucleotide sequence of miR92a-3p and the 3'-untranslated r e g i o n s (UTRs) of ADAMTS-4 and ADA M T S - 5 $\mathrm{m}$ R N A . b. 293T cells were

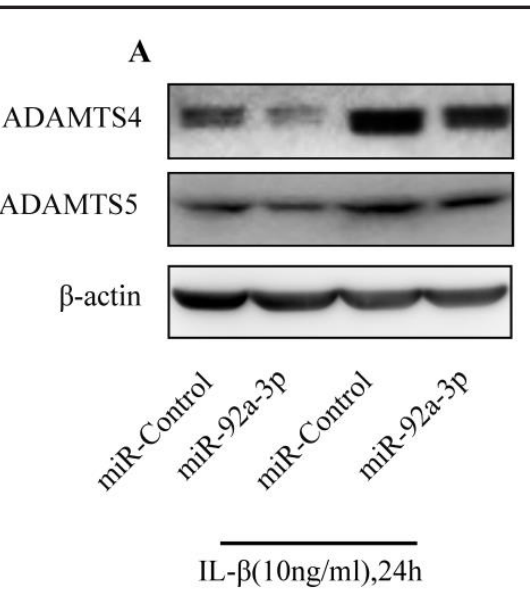

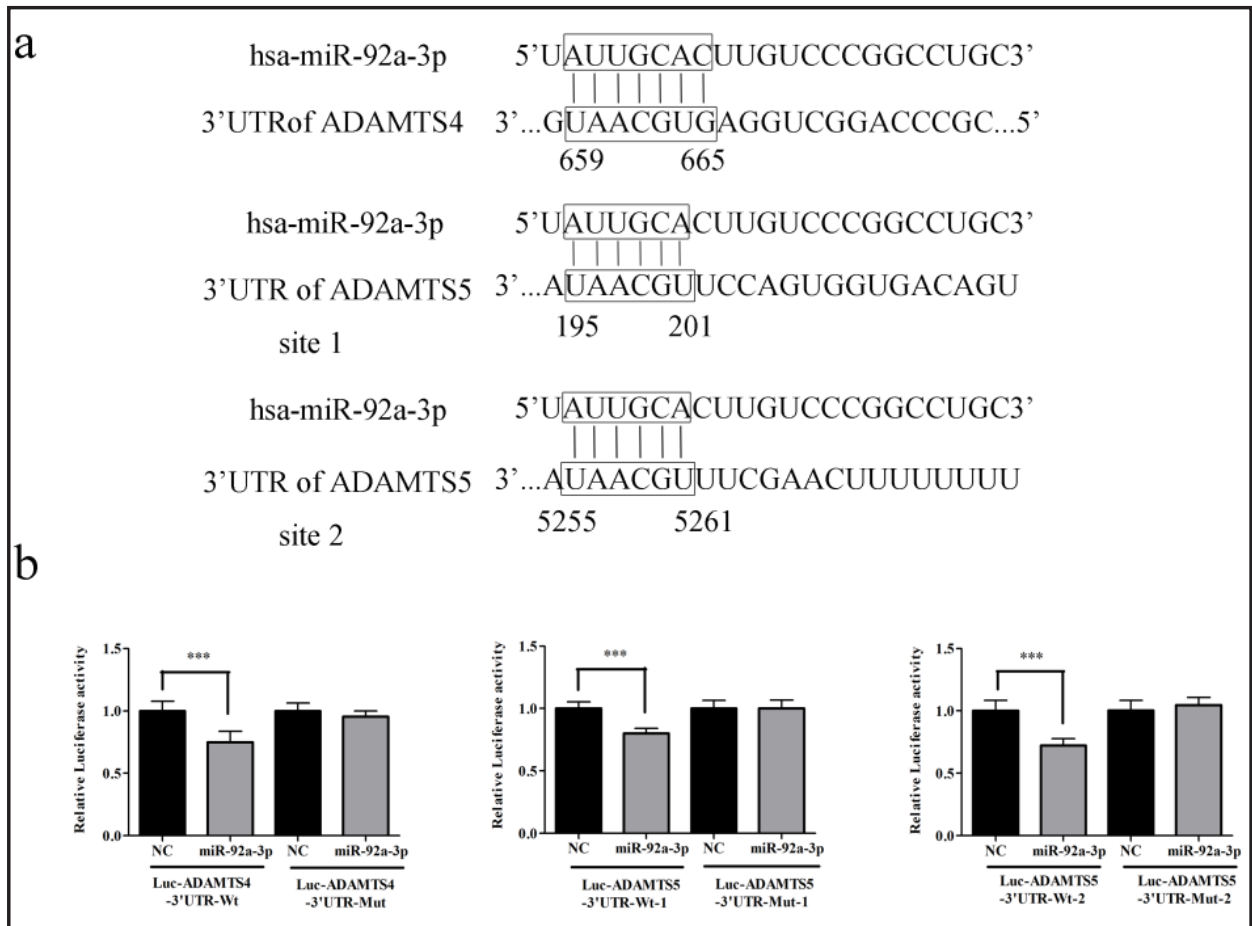

transfected with luciferase reporters carrying the wild-type 3'-UTRs of ADAMTS-4, ADAMTS-5 site 1, or ADAMTS-5 site 2 (Luc-ADAMTS4-UTR, Luc-ADAMTS5-site1-UTR, and Luc-ADAMTS5-site2-UTR, respectively), or with vectors containing mutated 3'-UTR sequences in which the putative miR-92a-3p-binding site was altered (Luc-ADAMTS4-UTR-mut, Luc-ADAMTS5-site1-UTR-mut, and Luc-ADAMTS5-site2-UTR-mut, respectively) or in combination with miR-control or miR-92a-3p, respectively. Cells were harvested after $24 \mathrm{~h}$, and luciferase activity was analyzed. Data are presented as means \pm standard deviations from three independent experiments. ${ }^{* * *} \mathrm{P}<0.001$.

protein expression in PHCs (Fig. 5I, J, and Fig.6B). These results suggested that ADAMTS-4/5 expression is downregulated by miR-92a-3p in human chondrocytes. 


\section{Cellular Physiology Cell Physiol Biochem 2017;44:38-52

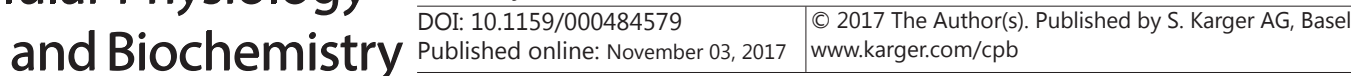 \\ Guping et al.: Role of Mir-92a-3p in Chondrogenesis and Degeneration}

miR-92a-3p directly targets ADAMTS-4 and ADAMTS-5 by interaction with the $3^{\prime}$-UTR

On the basis of bioinformatics analysis with TargetScan and micro.RNA.org, ADAMTS-4 and ADAMTS- 5 were identified as potential targets of miR-92a-3p. Furthermore, the qRTPCR data demonstrated that ADAMTS-4/5 were regulated by miR-92a-3p. We therefore utilized a luciferase reporter assay to further examine whether the $3^{\prime}$-UTRs of ADAMTS-4/5 contain a sequence capable of interacting with miR-92a-3p. For these analyses, 293T cells were transfected with luciferase reporters containing wild-type or mutated 3'-UTRs from ADAMTS-4, ADAMTS- 5 site 1, and ADAMTS- 5 site 2 in the presence or absence of miR-92a-3p or the negative control. The putative miR-92a-3p-binding sites for the ADAMTS-4/5 mRNA molecules are shown in Fig. 7a. Notably, cells co-transfected with miR-92a-3p and vectors encoding the wild-type 3 '-UTR sequences exhibited significantly lower luciferase activity than those co-transfected with the negative control and wild-type vectors, respectively. In contrast, there was no significant change in luciferase activity in cells co-transfected with miR-92a-3p and vectors in which the predicted miR-92a-3p-binding sites within the $3^{\prime}$ UTRs of ADAMTS- $4 / 5$ were mutated (Fig. $7 b$ ). Together, these data indicate that miR-92a-3p does indeed modulate ADAMTS-4/5 expression by binding to the 3 '-UTR of the respective sequences.

\section{Discussion}

It is widely accepted that ADAMTS-4/5 overexpression can play a key role in the destruction of cartilage in chronic joint diseases such as OA or rheumatoid arthritis (RA) [11-13, 15-17, 34-37]. In this study, we investigated miRNAs that are expressed in a differentiation-dependent pattern in hMSCs and articular chondrocytes. The combination of our previous research [27] and present results revealed that both miR-92a-3p and ADAMTS-4/5 have opposing expression patterns during the chondrogenic differentiation of hMSCs, especially during the late stage of chondrogenic differentiation, and that miR-92a-3p regulates ADAMTS- $4 / 5$ expression during chondrogenesis. We also demonstrated changes in the expression of miR-92a-3p in the cartilage of OA patients and in response to IL-1 $\beta$ stimulation. Specifically, miR-92a-3p expression was significantly decreased in OA cartilage compared with that in normal cartilage. The overexpression of miR-92a-3p suppressed IL-1 $\beta$-induced ADAMTS-4/5 production, while transfection with anti-miR-92a-3p notably increased their production levels. Moreover, we demonstrated that ADAMTS-4/5, critical proteinases in OA pathogenesis, are regulated by miR-92a-3p. Mutation of the miR-92a-3pbinding site in the 3'-UTRs of ADAMTS-4/5 mRNA led to complete abrogation of the miR92a-3p-mediated repression of reporter activity, indicating that ADAMTS-4/5 are targets for miR-92a-3p-mediated repression.

Recent studies have reported the role of specific miRNAs in the pathogenesis of various diseases. Matsukawa et al. [18] reported that miR-125b acts as a direct regulator of ADAMTS-4 expression in human OA chondrocytes, and that the reduction of miR-125b expression in OA cartilage in response to IL- $1 \beta$ is closely related to enhanced production of ADAMTS-4. Moreover, it has been demonstrated that hsa-miR-15a exerts protective effects against OA by targeting ADAMTS-5 in human chondrocytes [19].

MiR-92a is a component of the mir-17-92 cluster, which possesses oncogenic activity and can influence cell proliferation and tumorigenesis [38, 39]. Ning et al. [40] demonstrated a positive effect of miR-92a on the proliferation, differentiation, and survival of chondrogenic progenitor cells by targeting nog3, an inhibitor of the BMP signaling pathway. MiR-92a expression decreases rapidly in macrophages upon stimulation with Toll-like receptor ligands, and miR-92a controls the inflammatory response by targeting the MKK4/JNK/c-Jun pathway [41]. In our previous studies, we demonstrated that miR-92a-3p plays an important role in both cartilage development and degeneration via directly targeting HDAC2, and that siHDAC2 can also repress the expression of ADAMTS-4/5 in primary human chondrocytes $[26,27]$. 


\section{Cellular Physiology Cell Physiol Biochem 2017;44:38-52 and BiOChemistry \begin{tabular}{l|l} 
DOI: 10.1159/000484579 & $\begin{array}{l}\text { C } 2017 \text { The Author(s). Published by S. Karger AG, Basel } \\
\text { www.karger.com/cpb }\end{array}$
\end{tabular} \\ Guping et al.: Role of Mir-92a-3p in Chondrogenesis and Degeneration}

Specifically, in our previous study [27], when hMSCs were induced to differentiate into chondrocytes, miR-92a-3p expression was upregulated, especially during the early stage of chondrogenesis (days 14-21), but was subsequently down-regulated during hypertrophy and endochondral ossification (days 21-35) [27]. This expression pattern was quite similar to that of miR-140, which has been shown to stimulate chondrogenesis in vitro. In the present study, when hMSCs were induced to undergo chondrogenesis, overexpression of miR-92a-3p markedly suppressed ADAMTS-4/5 production and was associated with increased aggrecan expression, while suppression of miR-92a-3p enhanced ADAMTS-4/5 expression and was associated with reduced aggrecan expression [27]. Overall, these data suggest that miR-92a$3 p$ regulates ADAMTS- $4 / 5$ expression during chondrogenesis.

IL-1 $\beta$ is an important regulator of joint inflammation and cartilage degradation in OA, and the present findings provide a link between IL-1 $\beta$ and miR-92a-3p. In OA cartilage, decreased miR-92a-3p expression and a concomitant increase in ADAMTS-4/5 expression were observed in our previous study [27]. Furthermore, when PHCs were stimulated with IL-1 $\beta$, the expression of miR-92a-3p was significantly downregulated, similar to the effects observed with miR-125b [18] and miR-140 [25], which have been shown to directly regulate the expression of ADAMTS-4 and ADAMTS-5, respectively, and enhanced production of ADAMTS-4/5 protein was noted. Our results also suggested that miR-92a-3p targets the seed sites of human ADAMTS-4 (nucleotide position 659-665) and ADAMTS-5 (nucleotide positions 195-201 and 5255-5261). This observation, along with the results of the luciferase reporter assay, indicates that miR-92a-3p probably interacts with the $3^{\prime}$-UTRs of ADAMTS-4/5 mRNA and downregulates their expression at the post-transcriptional level. In addition, in our study, the IL-1 $\beta$-induced suppression of miR-92a-3p was reversed with treatment of NF- $\kappa B$ and MAPK inhibitors. A similar negative regulation of ADAMTS-4/5 in chondrocytes by activated NF- $\kappa B$ and MAPKs via miRNAs has been reported previously [4246], suggesting that miRNAs with similar functions in inflammation and degeneration may be regulated by a common set of catabolic pathways in chondrocytes, including NF- $\kappa \mathrm{B}$ and MAPK.

The present study has some limitations. First, we did not elucidate the in vivo significance of miR-92a-3p. Second, the detailed signaling pathways involved in IL-1 $\beta$-induced miR-92a$3 p$ inhibition and the potential of miR-92a-3p as an OA marker remain to be investigated. Moreover, the results of the differential expression of miR-92a-3p were observed in a small sample $(n=8)$. A study involving a larger sample size is necessary to validate these findings.

In summary, we found that miR-92a-3p is downregulated by IL- $1 \beta$, and may function as an important regulator of ADAMTS-4/5 in both chondrogenesis and OA pathogenesis. Further studies on miR-92a-3p have the potential to reveal important new regulatory pathways that control cartilage development and homeostasis and can provide the basis for novel therapeutic and/or preventive approaches for OA.

\section{Abbreviations}

ADAMTS4 (ADAM metallopeptidase with thrombospondin type 1 motif 4); ADAMTS5 (ADAM metallopeptidase with thrombospondintype 1 motif 5); qRT-PCR (quantitative reverse-transcription polymerase chain reaction).

\section{Acknowledgments}

We thank Xuerong Li, Caiqin Wang, and Mei Shang (Department of Parasitology, Zhongshan School of Medicine, Sun Yat-sen University, Guangzhou, China) for their aid with microscopy and other techniques. This study was supported by the National Nature Science Foundation of China (81371941, 81572119, 81472101 and 81672198) and the Guangdong Provincial Natural Science Foundation of China (201605122119317, 2013B021800295 and 


\section{Cellular Physiology Cell Physiol Biochem 2017;44:38-52

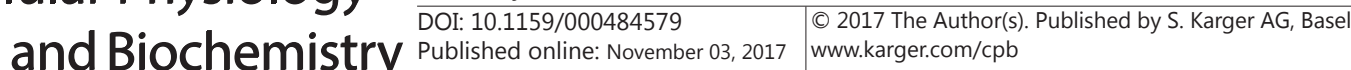

Guping et al.: Role of Mir-92a-3p in Chondrogenesis and Degeneration

2015A020212015). Study conception and design: Yan Kang, Yukang Li and Weiming Liao, Acquisition, analysis and interpretation of data: Guping Mao and Peihui Wu. Manuscript drafting and editing: Guping Mao. Critical revision of the article for important intellectual content: Ziji Zhang, Zhiqi Zhang, Weiming Liao, Yukang Li and Yan Kang. All authors approved the final version of the manuscript.

\section{Disclosure Statement}

Authors have no conflicts of interest to declare. Financial support had no impact on the outcome of this study.

\section{References}

1 Loeser RF, Goldring SR, Scanzello CR, Goldring MB: Osteoarthritis: A disease of the joint as an organ. Arthritis Rheum 2012;64:1697-1707.

2 Bijlsma JW, Berenbaum F, Lafeber FP: Osteoarthritis: An update with relevance for clinical practice. Lancet 2011;377:2115-2126.

3 Lane NE, Brandt K, Hawker G, Peeva E, Schreyer E, Tsuji W, Hochberg MC: OARSI-FDA initiative: Defining the disease state of osteoarthritis. Osteoarthritis Cartilage 2011;19:478-482.

4 Kobayashi M, Squires GR, Mousa A, Tanzer M, Zukor DJ, Antoniou J, Feige U, Poole AR: Role of interleukin-1 and tumor necrosis factor alpha in matrix degradation of human osteoarthritic cartilage. Arthritis Rheum 2005;52:128-135.

5 Daheshia M, Yao JQ: The interleukin 1beta pathway in the pathogenesis of osteoarthritis. J Rheumatol 2008;35:2306-2312.

6 Goldring MB: Osteoarthritis and cartilage: The role of cytokines. Curr Rheumatol Rep 2000;2:459-465.

-7 Fukui T, Tenborg E, Yik JH, Haudenschild DR: In-vitro and in-vivo imaging of MMP activity in cartilage and joint injury. Biochem Biophys Res Commun 2015;460:741-746.

8 Wang M, Sampson ER, Jin H, Li J, Ke QH, Im HJ, Chen D: MMP13 is a critical target gene during the progression of osteoarthritis. Arthritis Res Ther 2013;15:R5.

9 Sandy JD: A contentious issue finds some clarity: On the independent and complementary roles of aggrecanase activity and MMP activity in human joint aggrecanolysis. Osteoarthritis Cartilage 2006;14:95100.

10 Tetsunaga T, Nishida K, Furumatsu T, Naruse K, Hirohata S, Yoshida A, Saito T, Ozaki T: Regulation of mechanical stress-induced MMP-13 and ADAMTS- 5 expression by RUNX-2 transcriptional factor in SW1353 chondrocyte-like cells. Osteoarthritis Cartilage 2011;19:222-232.

11 Yatabe T, Mochizuki S, Takizawa M, Chijiiwa M, Okada A, Kimura T, Fujita Y, Matsumoto H, Toyama Y, Okada Y: Hyaluronan inhibits expression of ADAMTS4 (aggrecanase-1) in human osteoarthritic chondrocytes. Ann Rheum Dis 2009;68:1051-1058.

12 Tortorella MD, Malfait AM, Deccico C, Arner E: The role of ADAM-TS4 (aggrecanase-1) and ADAM-TS5 (aggrecanase-2) in a model of cartilage degradation. Osteoarthritis Cartilage 2001;9:539-552.

13 Jones GC, Riley GP: ADAMTS proteinases: A multi-domain, multi-functional family with roles in extracellular matrix turnover and arthritis. Arthritis Res Ther 2005;7:160-169.

14 Yang CY, Chanalaris A, Troeberg L: ADAMTS and ADAM metalloproteinases in osteoarthritis - looking beyond the 'usual suspects'. Osteoarthritis Cartilage 2017;25:1000-1009.

15 Majumdar MK, Askew R, Schelling S, Stedman N, Blanchet T, Hopkins B, Morris EA, Glasson SS: Doubleknockout of ADAMTS-4 and ADAMTS-5 in mice results in physiologically normal animals and prevents the progression of osteoarthritis. Arthritis Rheum 2007;56:3670-3674.

16 Song RH, Tortorella MD, Malfait AM, Alston JT, Yang Z, Arner EC, Griggs DW: Aggrecan degradation in human articular cartilage explants is mediated by both ADAMTS- 4 and ADAMTS- 5 Arthritis Rheum 2007;56:575-585. 


\section{Cellular Physiology Cell Physiol Biochem 2017;44:38-52 \begin{tabular}{l|l} 
and Biochemistry Published online: November 03, 2017 & $\begin{array}{l}\text { (c) } 2017 \text { The Author(s). Published by S. Karger AG, Basel } \\
\text { www.karger.com/cpb }\end{array}$
\end{tabular}}

Guping et al.: Role of Mir-92a-3p in Chondrogenesis and Degeneration

17 Naito S, Shiomi T, Okada A, Kimura T, Chijiiwa M, Fujita Y, Yatabe T, Komiya K, Enomoto H, Fujikawa K, Okada Y: Expression of ADAMTS4 (aggrecanase-1) in human osteoarthritic cartilage. Pathol Int 2007;57:703-711.

18 Matsukawa T, Sakai T, Yonezawa T, Hiraiwa H, Hamada T, Nakashima M, Ono Y, Ishizuka S, Nakahara H, Lotz MK, Asahara H, Ishiguro N: MicroRNA-125b regulates the expression of aggrecanase-1 (ADAMTS-4) in human osteoarthritic chondrocytes. Arthritis Res Ther 2013;15:R28.

19 Lu X, Lin J, Jin J, Qian W, Weng X: Hsa-miR-15a exerts protective effects against osteoarthritis by targeting aggrecanase-2 (ADAMTS5) in human chondrocytes. Int J Mol Med 2016;37:509-516.

-20 Miyaki S, Nakasa T, Otsuki S, Grogan SP, Higashiyama R, Inoue A, Kato Y, Sato T, Lotz MK, Asahara H: MicroRNA-140 is expressed in differentiated human articular chondrocytes and modulates interleukin-1 responses. Arthritis Rheum 2009;60:2723-2730.

21 Lim LP, Lau NC, Garrett-Engele P, Grimson A, Schelter JM, Castle J, Bartel DP, Linsley PS, Johnson JM: Microarray analysis shows that some microRNAs downregulate large numbers of target mRNAs. Nature 2005;433:769-773.

22 Bartel DP: MicroRNAs: Genomics, biogenesis, mechanism, and function. Cell 2004;116:281-297.

-23 Zhang Z, Hou C, Meng F, Zhao X, Zhang Z, Huang G, Chen W, Fu M, Liao W: MiR-455-3p regulates early chondrogenic differentiation via inhibiting Runx2 FEBS Lett 2015;589:3671-3678.

24 Chen W, Chen L, Zhang Z, Meng F, Huang G, Sheng P, Zhang Z, Liao W: MicroRNA-455-3p modulates cartilage development and degeneration through modification of histone H3 acetylation. Biochim Biophys Acta 2016;1863:2881-2891.

25 Miyaki S, Sato T, Inoue A, Otsuki S, Ito Y, Yokoyama S, Kato Y, Takemoto F, Nakasa T, Yamashita S, Takada S, Lotz MK, Ueno-Kudo H, Asahara H: MicroRNA-140 plays dual roles in both cartilage development and homeostasis. Genes Dev 2010;24:1173-1185.

-26 Zhang Z, Kang Y, Zhang Z, Zhang H, Duan X, Liu J, Li X, Liao W: Expression of microRNAs during chondrogenesis of human adipose-derived stem cells. Osteoarthritis Cartilage 2012;20:1638-1646.

-27 Mao G, Zhang Z, Huang Z, Chen W, Huang G, Meng F, Zhang Z, Kang Y: MicroRNA-92a-3p regulates the expression of cartilage-specific genes by directly targeting histone deacetylase 2 in chondrogenesis and degradation. Osteoarthritis Cartilage 2017;25:521-532.

28 Meng FG, Zhang ZQ, Huang GX, Chen WS, Zhang ZJ, He AS, Liao WM: Chondrogenesis of mesenchymal stem cells in a novel hyaluronate-collagen-tricalcium phosphate scaffolds for knee repair. Eur Cell Mater 2016;31:79-94.

29 Zhang L, Su P, Xu C, Yang J, Yu W, Huang D: Chondrogenic differentiation of human mesenchymal stem cells: A comparison between micromass and pellet culture systems. Biotechnol Lett 2010;32:1339-1346.

-30 Hou C, Zhang Z, Zhang Z, Wu P, Zhao X, Fu M, Sheng P, Kang Y, Liao W: Presence and function of microRNA92a in chondrogenic ATDC5 and adipose-derived mesenchymal stem cells. Mol Med Rep 2015;12:48774886.

31 Hou C, Meng F, Zhang Z, Kang Y, Chen W, Huang G, Fu M, Sheng P, Zhang Z, Liao W: The role of MicroRNA-381 in chondrogenesis and interleukin-1-beta induced chondrocyte responses. Cell Physiol Biochem 2015;36:1753-1766.

-32 Meng F, Zhang Z, Chen W, Huang G, He A, Hou C, Long Y, Yang Z, Zhang Z, Liao W: MicroRNA-320 regulates matrix metalloproteinase-13 expression in chondrogenesis and interleukin-1beta-induced chondrocyte responses. Osteoarthritis Cartilage 2016;24:932-941.

33 Ronald L. Wasserstein, Nicole A. Lazar: The ASA's statement on p-values: context, process, and purpose.The American Statistician 2016 DOI:10.1080/00031305.

-34 Verma P, Dalal K: ADAMTS-4 and ADAMTS-5: Key enzymes in osteoarthritis. J Cell Biochem 2011;112:35073514.

-35 Karsenty G: An aggrecanase and osteoarthritis. N Engl J Med 2005;353:522-523.

-36 Glasson SS, Askew R, Sheppard B, Carito B, Blanchet T, Ma HL, Flannery CR, Peluso D, Kanki K, Yang Z, Majumdar MK, Morris EA: Deletion of active ADAMTS5 prevents cartilage degradation in a murine model of osteoarthritis. Nature 2005;434:644-648.

-37 Botter SM, Glasson SS, Hopkins B, Clockaerts S, Weinans H, van Leeuwen JP, van Osch GJ: ADAMTS5-/mice have less subchondral bone changes after induction of osteoarthritis through surgical instability: Implications for a link between cartilage and subchondral bone changes. Osteoarthritis Cartilage 2009;17:636-645. 


\section{Cellular Physiology Cell Physiol Biochem 2017;44:38-52

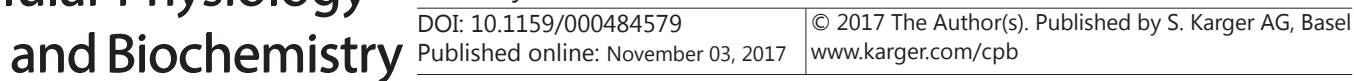 \\ Guping et al.: Role of Mir-92a-3p in Chondrogenesis and Degeneration}

-38 He L, Thomson JM, Hemann MT, Hernando-Monge E, Mu D, Goodson S, Powers S, Cordon-Cardo C, Lowe SW, Hannon GJ, Hammond SM: A microRNA polycistron as a potential human oncogene. Nature 2005;435:828833.

39 O’Donnell KA, Wentzel EA, Zeller KI, Dang CV, Mendell JT: C-Myc-regulated microRNAs modulate E2F1 expression. Nature 2005;435:839-843.

40 Ning G, Liu X, Dai M, Meng A, Wang Q: MicroRNA-92a upholds Bmp signaling by targeting noggin3 during pharyngeal cartilage formation. Dev Cell 2013;24:283-295.

-41 Lai L, Song Y, Liu Y, Chen Q, Han Q, Chen W, Pan T, Zhang Y, Cao X, Wang Q: MicroRNA-92a negatively regulates Toll-like receptor (TLR)-triggered inflammatory response in macrophages by targeting MKK4 kinase. J Biol Chem 2013;288:7956-7967.

42 Chockalingam PS, Varadarajan U, Sheldon R, Fortier E, LaVallie ER, Morris EA, Yaworsky PJ, Majumdar MK: Involvement of protein kinase Czeta in interleukin-1beta induction of ADAMTS-4 and type 2 nitric oxide synthase via NF-kappaB signaling in primary human osteoarthritic chondrocytes. Arthritis Rheum 2007;56:4074-4083.

43 Tian Y, Yuan W, Fujita N, Wang J, Wang H, Shapiro IM, Risbud MV: Inflammatory cytokines associated with degenerative disc disease control aggrecanase-1 (ADAMTS-4) expression in nucleus pulposus cells through MAPK and NF-kappaB. Am J Pathol 2013;182:2310-2321.

44 Saito T, Nishida K, Furumatsu T, Yoshida A, Ozawa M, Ozaki T: Histone deacetylase inhibitors suppress mechanical stress-induced expression of RUNX-2 and ADAMTS- 5 through the inhibition of the MAPK signaling pathway in cultured human chondrocytes. Osteoarthritis Cartilage 2013;21:165-174.

-45 Wang J, Markova D, Anderson DG, Zheng Z, Shapiro IM, Risbud MV: TNF-alpha and IL-1beta promote a disintegrin-like and metalloprotease with thrombospondin type I motif-5-mediated aggrecan degradation through syndecan-4 in intervertebral disc. J Biol Chem 2011;286:39738-39749.

-46 Zhang F, Wang J, Chu J, Yang C, Xiao H, Zhao C, Sun Z, Gao X, Chen G, Han Z, Zou W, Liu T: MicroRNA-146a induced by hypoxia promotes chondrocyte autophagy through bcl-2 Cell Physiol Biochem 2015;37:1442-1453. 